Voix et Images

\title{
L’idéologie du pays dans le roman québécois contemporain : Il n'y a pas de pays sans grand-père et l'intertexte national
}

\section{Jacques Allard}

Volume 5, numéro 1, automne 1979

Jacques Godbout

URI : https://id.erudit.org/iderudit/200191ar

DOI : https://doi.org/10.7202/200191ar

Aller au sommaire du numéro

Éditeur(s)

Les Presses de l'Université du Québec

ISSN

0318-9201 (imprimé)

1705-933X (numérique)

Découvrir la revue

Citer cet article

Allard, J. (1979). L'idéologie du pays dans le roman québécois contemporain : Il n'y a pas de pays sans grand-père et l'intertexte national. Voix et Images, 5(1),

117-132. https://doi.org/10.7202/200191ar d'utilisation que vous pouvez consulter en ligne. 
L'Idéologle du pays dans

le roman québécols contemporain:

\section{II n'y a pas de pays sans grand-père et l'intertexte national}

L'imagination québécoise n'a pas cessé, depuis la chute de la NouvelleFrance en 1760, de rêver sur le pays perdu et de travailler à sa reconquête lente et rusée. Le thème même du pays, à travers différents motifs, s'est manifesté dans toute notre littérature. Des historiens, des sociologues et des critiques littéraires ont régulièrement, au fil des époques, analysé le phénomène, allant souvent jusqu'à provoquer eux-mêmes sa floraison. Mais on ne connaît pas encore d'analyse littéraire qui en aurait étudié la figure, de ses premières manifestations jusqu'à celles que nous connaissons aujourd'hui.

Je n'ai pas l'intention de répondre dès maintenant à ce grand défi qui consisterait à mettre en place toute l'imagerie nationale dans sa représentation littéraire. II s'agit pour moi d'une recherche en cours et dont je ne vois pas encore la fin. Je me contenterai, pour l'instant de poser les jalons d'une problématique, telle qu'elle surgit dans un roman récent qui se propose lui-même comme une synthèse de l'intertexte national, le roman de Roch Carrier, II n'y a pas de pays sans grand-père.

Comment opérer la saisie idéologique de la figure du pays ? Plutôt que de commencer par une définition théorique de ma procédure, j'irai droit au texte pour en donner graduellement une illustration, me contentant, pour lors, de préciser que par "idéologie" j'entends le système de représentations tel que défini assez couramment. J'ajouterai que par le mot «pays" je retiens ce qu'en dit le dictionnaire: le sens premier est celui du territoire habité par une collectivité et constituant par là une réalité géographique nationale, provinciale ou régionale; le pays, quand il s'y ajoute une notion d'appartenance renvoie aussi à la patrie, petite ou grande, celle du village, celle des grands ancêtres ou celle, plus simplement, de la terre d'élection; c'est aussi, enfin, une région géographique vue dans son aspect strictement physique: c'est la contrée, le terrain ou, par métaphore, le domaine, le royaume.

Comme on le voit, le pays est un mot-réseau, une sorte d'échangeur à voies multiples dont le discours littéraire, d'où qu'il soit, joue constamment, quelles que soient les contingences géo-politiques vécues par une collectivité sur un territoire donné. On voudra donc bien se souvenir que l'exploration 
que je me propose finalement d'accomplir ne trouvera ici que des éléments de repérage dans cette imagination que nous Québécois avons d'un espace vécu et rêvé.

\section{Le titre et le conditionnement textuel}

/I n'y a pas de pays sans grand-père a été publié en 1977 '. C'est le sixième roman de cet auteur à succès et il annonce dès son titre la couleur paysanne que l'on connaît depuis La guerre, yes sir! (68), Floralie où es-tu? (69), Il est par là le soleil (70) et le Jardin des délices (75). (Je laisse de côté les contes qui viennent de paraître, Les enfants du bonhomme dans la lune). Et si l'on veut faire place, dans ce tableau, au roman "urbain" intitulé $L \theta$ deux-millième étage (73), il suffira de rappeler l'échec du paysan Dorval venu s'établir en ville: il n'aspire qu'à retourner à la campagne. Ainsi, I/ n'y a pas de pays sans grand-père paraît bien nous ramener à la Beauce natale, essentielle de son auteur.

Mais un examen plus attentif du conditionnement textuel (le titre et la couverture) permet déjà de préciser ce qu'on pourrait nommer l'annonce idéologique ${ }^{2}$. Ceux qu'intéressent les marques de l'institution littéraire ne seront sans doute pas indifférents à l'importance donnée par la grosseur des caractères au nom de l'auteur: l'éditeur "vend" en effet davantage l'auteur que son livre. Mais la force assertive du titre, posé sur l'image d'un village est tout de même très éloquente. Cette affirmation tranchée, similaire à celle si déictique du roman précédent (II est par là le soleil) découvre aisément un "programme": nous avons là la conclusion d'une démonstration déjà faite, proche en cela du premier titre du cycle: La guerre, yes sir! où la ponctuation populaire retentissait.

A celui qu'étonnerait cette mise en train analytique, qui y verrait un hors d'œuvre, un curieux détour par le hors-texte, je signalerai que l'allure programmatique de la présentation extérieure de ce roman se confirme aisément en couverture arrière où l'éditeur cite un extrait du texte, le donnant comme les vers d'une comptine nettement patriotique:

Nous marcherons sur la terre

où ton père a marché

avant qu'il ne soit terrassé par le désespoir,

où mon père a marché,

et son père avant lui,

et son grand-père;

nous pêcherons dans cette eau

où nos pères ont pêché

et nous chasserons dans cette forêt

où nos pères ont chassé,

toujours à la sauvette, à la façon

d'étrangers, en territoires interdits,

mais nous savons toi et moi,

que le bon Dieu a créé cette terre

pour nous et nos enfants... 
Le lecteur-consommateur ne peut donc s'interroger longtemps sur ce qui est ici mis en circulation ou mis en marché. C'est la terre, celle que l'on " marche», comme disent transitivement nos paysans, de père en fils, malgré les lâcheurs, malgré les interdits, malgré la dépossession mais avec la caution suprême, celle de Dieu. Voilà le programme de lecture qui est offert dans la reprise des mots clés: "ce n'était qu'un début, continuons le combat ", dit, en employant le futur, un vieil homme à son petit-fils. Dans ce message brut, c'est une figure nettement nationale qui est donnée au territoire non reconnu d'une collectivité, à partir du terrain rural ou du moins de la grande nature.

\section{La masse narrative et son découpage}

Cette annonce idéologique est-elle conforme au récit qui continue à la première page? Un narrateur y prononce d'abord un mot: "Attendre... " et donne la parole à Vieux-Thomas qui prend le relai. II a soixante-treize ans (tout en s'en donnant soixante-dix-sept comme pour mieux accentuer un isolement et se ronge d'impatience dans sa chaise berçante, paraissant attendre la délivrance et, surtout, le combat que lui apporterait la mort. Et le narrateur de reprendre la parole, expliquant que le vieux n'en peut plus de se bercer dans l'ennui de cette maison où règne la jeunesse... Et le texte continue de se dérouler, massif, occupant tout l'espace, sans indication de chapitre, ouvrant les parenthèses du souvenir, des voix entendues; laissant tout juste la place des points de suspension dans une ponctuation généralement précise; au rythme de la berceuse, de la jonglerie du vieux dans sa chaise, comme l'illustration parfaite du mouvement perpétuel (intérieur) dans l'immobilité. L'effet produit par cette mise en scène textuelle est celui d'un appui incontestable à la valeur affirmative du titre.

Voilà pourquoi il faut stopper ce déroulement narratif, en dépecer la masse pour voir comment s'établit la force de l'apparente démonstration, la pertinence de ce «théorème" littéraire. De fait, le narré se partage aisément en trois parties si l'on privilégie le fil événementiel qui projettera le vieux hors de sa chaise pour l'immobiliser à nouveau dans un asile de vieillards, le faisant passer de sa chaise berçante à la camisole de force terminale.

\section{La complainte du vieux}

La première partie (une trentaine de pages) met en place les ruminations du grand-père bloqué dans sa propre maison régentée par la famille de l'un de ses fils, Dieudonné: en fait c'est la Bru et les petits-enfants qui mènent, le fils au nom prédestiné constituant pour son père une véritable "épreuve"... Le vieil homme est donc tout entier livré à ses souvenirs, sourd à ce qui l'entoure si ce n'est à la télévision qui l'informe de l'actualité: détournement d'avion, famines, visite de la reine d'Angleterre au Québec, etc., tous événements qui le ramènent à ses souvenirs de jeunesse et à ses frustrations présentes. Son temps à lui était celui de la vraie vie alors que l'on travaillait, plutôt que d'être paresseux comme Dieudonné et ses fils. En ce 
temps-là, il a construit sa propre maison, gagné sa vie comme bûcheron, lutté à armes égales avec le chevreuil ou le poisson; il a aussi bâti sa chaise comme le mobilier, et même des cercueils: le sien, et ceux des enfants perdus. Aujourd'hui, tout cela lui est refusé, nié par ceux qui l'entourent: on lui confisque son couteau de chasse, ses hameçons, sa hache, ses lunettes. II est confiné à l'inaction, d'où sa longue lamentation et ses questions à son "cher bon Dieu".

II y a là dans ce monologue, débordant de métaphores et d'humour, un ensemble de données qui peuvent se ramener à la proposition suivante: il faut retrouver la nature, la foret primordiale, le combat fondamental, loyal, avec soi et les autres, animaux et hommes, et ainsi se construire : «... bâtir sa maison, c'est naître une deuxième fois" (p. 32) ; “construire sa berceuse... c'est bâtir sa deuxième ossature" (p. 14). Cette proposition première ${ }^{3}$ se donne sur le mode d'une complainte dont les nombreux couplets-récits du passé, touchants autant que redondants, sont entrecoupés du refrain de l'aujourd'hui aliénant. A ce propos, notons qu'on y retrouve la revendication écologique d'une certaine jeunesse (je pense aux groupes inspirés par Mainmise ${ }^{4}$ ) non représentée comme telle dans le texte. Et, au sujet du passé glorieux de Vieux-Thomas, il est aussi utile de souligner que nous avons affaire à un bûcheron-chasseur plutôt qu'à un cultivateur.

\section{Le chant de la réconciliatlon}

La deuxième partie vient ensuite étoffer longuement (près de soixantedix pages, soit les deux tiers du récit) la proposition initiale, cernant davantage les causes d'un état qui d'individuel, propre au vieux et à sa vision du monde présent, passe à celui de toute une collectivité. Cette croissance narrative en spirale repose sur le même jeu alternatif de la confrontation du passé et du présent. Mais cette alternative structurale se fera concaténation (enchaînement) et conjonction des deux temps, à l'occasion d'un événement : l'arrestation d'un petit-fils dont on n'a pas entendu parler jusque-là. JeanThomas a été arrêté lors d'une manifestation contre la reine en visite dans sa bonne ville de Québec.

Résumons sommairement la procédure narrative. La reprise des motifs et des thèmes de la première partie se produit par le biais de très nombreux micro-récits: la mort du père de Vieux-Thomas (foudroyé par l'éclair, il est mort debout, et non dans une chaise); celle de l'épouse, la Défunte dont le vieux conteur n'arrive pas à retrouver le nom; une histoire "vécue" de l'existence du diable; la pêche dans le lac volé par les Anglais (sans doute américains); la visite des Plaines d'Abraham (Vieux-Thomas promené dans la Cadillac d'un de ses fils, chauffeur de grand-patron); la " tranquillisation " de Dieudonné qui comme Menaud a refusé de livrer "sa " forêt aux Anglais et en est resté "dérangé"; la longue marche du jeune Vieux-Thomas vers les chantiers de la Mauricie, aventure initiatique comme son voyage au bout du monde et de la nuit dans la chaufferie d'un cargo, pendant la crise des années trente. 
Tous ces récits-couplets en viennent donc à s'articuler graduellement au vécu présent de la maison où Vieux-Thomas “jongle». En effet, l'arrestation de Jean-Thomas déclenche les interventions épisodiques des petitsenfants qui rappellent l'histoire du Québec et font au passage le procès de l'État et du Pouvoir. Pendant ce temps, le grand-père, réputé ne pas entendre, finit par comprendre le geste du petit-fils préféré, sa mémoire lui ramenant le souvenir des lectures que lui faisait Jean-Thomas. De telle sorte que le refrain du grand-père se modifie peu à peu pendant qu'il veille en méditant sur le sort de l'emprisonné. Sa jonglerie culmine en des pages où s'établit le programme des retrouvailles futures, le chant des générations réconciliées.

C'est alors que le "théorème" se précise, si l'on veut bien se souvenir que le théorème est fait d'une proposition qui résulte d'autres propositions déjà posées. Voici ce que projette, dans l'ordre, Vieux-Thomas quand il retrouvera son petit-fils: 10 l'échange des récits et des temps, le partage de l'expérience et de la jeunesse, celui de la tradition et du présent; tout cela contre l'asservissement collectif et ses rois-nègres (policiers, juges et autres gens de pouvoir); $2^{\circ}$ la marche de la terre comme territoire primordial (c'est le texte de la couverture arrière), la reconquête des forêts du passé dans l'espace balisé par les générations antérieures; $3^{\circ}$ l'apprentissage d'une langue d'amour et non de soumission (que serait l'anglais), ce sera la langue de la nature; $4^{0}$ refaire les lectures rituelles, c'est-à-dire reprendre les propositions de Louis Hémon dans Maria Chapdelaine, telles que reprises dans Menaud maitre-draveur.

\section{La sortie de Vleux-Thomas}

La dernière partie peut être établie à partir du moment où le grand-père commence les préparatifs de son coup. II n'est pas question d'un coup d'État, évidemment. Que peut faire un vieux beauceron, politisé sur le tard et confiné à sa télé ? Détourner un avion? Celui qui va de son village à Québec n'a pas d'ailes comme le dit le narrateur: c'est l'autobus qui s'arrête près de l'église où le vieux a ses habitudes matinales. Or, le lendemain de sa longue veille, le grand-père se jugeant d'ailleurs appuyé par son «cher bon Dieu " (il lui parle constamment) monte dans l'autobus plutôt que d'aller à la messe. II menace le conducteur de son couteau de chasse et le force à le conduire directement à la prison de Jean-Thomas. Le voyage sera encore l'occasion de rappels du passé, en particulier du retour de Justine à son village natal et de la victoire sur son mari anglophone, dûment écrasé par le fils d'Aristote (homme fort de l'ancien temps...). II y a aussi le souvenir des champs à "dérocher" et dont l'État veut le reboisement, niant par là le travail même des ancêtres. Et puis les inquiétudes: par exemple, Jean-Thomas ne pourra enlever les pierres en criant ou en manifestant. Ainsi, la narration, toujours plus intéressée aux ruminations du vieux ne s'attardera pas au voyage qui ne sera interrompu qu'une fois par une astuce non convaincante du conducteur. La route reprendra qui suivra les souvenirs et la rêverie du pirate de terre. Et cette équipée beauceronne (on pense au saccage récent du Centre commercial de 
Saint-Georges-de-Beauce par un amoureux déçu) prendra fin alors que, tout à sa songerie, le vieux sera immobilisé par des mains aussi soudaines qu'inconnues, plongé dans une camisole de force et enfermé dans une maison de vieillards où il attendra la délivrance par Jean-Thomas, disant: "J'veux mourir libre" (p. 116). Ce seront les derniers mots du récit.

Dans quelle mesure, cette dernière partie enrichit-elle le message si fortement accusé dans la partie précédente? Relevons sommairement les faits suivants: plutôt que de se contenter d'une prière (la messe), VieuxThomas a décidé de tenter de libérer son petit-fils par la violence, quitte à être réduit à son tour au cri, à la revendication de la liberté qui avait déjà causé l'emprisonnement de Jean-Thomas. A cette quête désespérée de liberté se sont mêlées d'autres images : celle de la réintégration de Justine à son village d'origine; celle de l'écrasement physique du mari "étranger" et celle de la terre à nettoyer plutôt qu'à reboiser. On trouve donc ici des valeurs déjà relevées plus haut, l'action violente étant elle-même reprise au compte du grand-père. Mais avant de faire la synthèse de toutes ces données glanées au long de notre découpage, il nous semble impératif de faire quelques mises au point sur le fonctionnement narratif et énonciatif de ce récit.

\section{Narration et énonciation}

Remarquons d'abord que ce qui intéresse ici la narration n'est pas vraiment l'argument, au sens dramatique, actantiel. On ne veut pas raconter comment un vieil homme parvient à détourner un car pour libérer un prisonnier politique. II s'agit de montrer plutôt comment le désir de liberté revient à un "pensionné", à un laisser-pour-compte du temps, de la famille et de la société. D'où la mise en place initiale de sa complainte nostalgique, puis celle de son long réveil activé par une actualité qui parvient à le rejoindre, cette politisation de la rumination le conduisant au geste apparemment absurde, sénile de la fin. Dans cette enfilade de micro-récits organisés en spirale, le grand cercle intermédiaire que constitue la deuxième partie nous paraît bien fonder l'étayage du programme repéré dans l'annonce idéologique.

Pourtant, ce discours narratif n'a pas besoin de la rhétorique de la preuve, de la démonstration du roman à thèse qu'on pouvait prévoir au départ. II n'en use pas. Le problème n'est pas posé puis résolu de façon systématique, dans une économie stricte des moyens employés. Nous n'avons pas davantage affaire à un pamphlet où les interlocuteurs sont nettement les protagonistes identifiés: locuteur et allocutaire, auteur et lecteur-cible. Et, cependant personne ne peut rester insensible à un message précis. Que se passe-t-il donc?

Nous sommes ici dans la structure du conte où le discours se donne en parole, celle d'un locuteur unique et privilégié, soit Vieux-Thomas. Sa parole est débordante, "naturelle» et non stratégique: les métaphores, les répétitions donnent à sa "parolade»5, comme dirait Bessette, ce tour littéraire 
qu'on pourrait caractériser par le trop-plein, l'itération poétique. Ainsi, sommes-nous passés, dans notre lecture, d'un repérage programmatique (à thèse) à la perception d'une complainte et d'un chant, ce qui suffirait à démontrer la facilité dangereusement réductrice d'une analyse rapide: un texte littéraire ne se réduit pas à un système de représentations aisément codifiables de thèmes, d'idées, d'images, de motifs, de figures. II faut en convenir. Mais il n'en reste pas moins qu'un ensemble de propositions puisse être dégagé et systématisé pour les fins limitées d'une analyse idéologique. Voilà pourquoi il est légitime de lire l'intention programmatique dans tout l'arrangement narratif et son énonciation.

Ainsi comment ne pas voir que la parole de Vieux-Thomas est continuellement soutenue, relayée par un narrateur-traducteur-écouteur qui vient à la rescousse du premier quand les mots lui manquent et à celle du lecteurdestinataire, qui autrement n'entendrait que ce qu'un vieux sourd veut bien percevoir et raconter de son cru. Il va de soi que ce relai énonciatif devient on ne peut plus précieux pour notre analyse, sans que l'on puisse affirmer par ailleurs qu'il introduise une dissonnance dans l'étalement de la parole du conteur premier. Fidèle à Vieux-Thomas, et à son registre discursif le narrateur nous permet tout de même une distanciation du grand parleur. Un exemple: la perception qu'a le vieux des petits-enfants qui l'entourent est relativement injuste, dans la mesure où leur vision historique est toute proche de la sienne propre.

Autre remarque à propos de l'énonciation: il y a dans ce marcottage narratif un interlocuteur bien présent, qui semble aller de soi et auquel VieuxThomas s'adresse en faisant lui-même questions et réponses. C'est le « cher bon Dieum. Ce recours au tout-puissant Lecteur joue un rôle phatique important que l'on pourrait qualifier de détourné: dans le conte traditionnel, le conteur interroge fréquemment son auditoire, sollicitant son attention, son assentiment. Et, ici, Vieux-Thomas n'arrive pas à rejoindre son proche auditoire familial auquel aurait pu éventuellement s'identifier le lecteur. Aussi, sans qu'il soit à la portée de tout le monde de se prendre pour le suprême Lecteur, l'on peut dire qu'on ne peut se sentir, à la longue, que sollicité et manipulé, nous simple lecteur, par les appels de Vieux-Thomas, puisque nous sommes aussi fait juge des plaintes et revendications du vieux et de son narrateur.

On comprendra donc mieux comment la narration et l'énonciation jouent un rôle et ont une fonction bien précise pour l'efficace idéologique. Ce texte de Carrier manifeste une volonté de démonstration dans l'organisation du narré et le libellé discursif. La machine tourne rondement dans un accord profond du propos et de la forme. Aussi faut-il se méfier d'un jugement comme celui-ci:

Vieux-Thomas n'est hélas qu'un grand-père à thèse. Émouvant parfois au détour d'une page, parce que tout de même Roch Carrier est un écrivain, un vrai; mais le plus souvent embourbé dans un magma idéologique dont la parole n'arrive pas à se déloger.

(Le Devoir, 18 juin 1977, p. 19) 
Ces lignes sont de Gilles Marcotte. Je n'ai pas réagi autrement à la première lecture. Mais un retour au texte m'a convaincu de l'injustesse de cette première vue. Le grand-père n'est pas qu' à thèse» et le magma n'est qu'apparent: cette masse textuelle se découpe et s'entend clairement pour qui tente d'en examiner l'architecture cachée.

\section{La flgure du pays}

Notre lecture permet de dégager maintenant les données fondamentales de la figure du pays dans le récit de Carrier. A partir du titre et de tout le conditionnement textuel, de l'allure programmatique initiale, en passant par l'étayage narratif et le soutien constant de son énonciation, se dégagent une image et des valeurs. Si nous revenons à la définition du dictionnaire, force nous est de reconnaître la prédominance de la notion d'appartenance d'un territoire précisément nommé et délimité.

Le pays est ici celui des ancêtres proches et lointains, la patrie. C'est d'abord la petite (natale, villageoise, beauceronne) qui s'inscrit ferme dans la parole directe et indirecte de Vieux-Thomas. Puis c'est la grande, le territoire non plus régional mais celui, collectif, si fréquemment dénommé "pays de Québec" et opposé, à plus d'une reprise à un État dominant, opressant qui, lui, n'est pas nommé. La grand patrie se construit progressivement dans les retrouvailles du grand-père et du petit-fils préféré, comme dans les conversations des autres enfants que la narration nous fait entendre. Cette double dimension patriotique se maintiendra jusqu'à la fin, à la faveur du fil narratif de l'emprisonnement et de la tentative de libération, fil qui permet de nouer les racines passées, présentes et futures des protagonistes, tout en proposant la réconciliation de la tradition et de la modernité. II va de soi que cette dernière n'est pas favorisée, dans la mesure où elle ne parvient au lecteur qu'à travers le filtre de l'ancien (le grand-père). Mais elle est là, sans qu'on puisse parler de révolution (opposée à la tradition), à moins qu'il ne s'agisse de la «tranquille révolution» comme l'a dit la presse anglocanadienne, en parlant de nos années soixante. On pourrait faire une nuance de même ordre en remarquant que les retrouvailles du grand-père et du petitfils sont purement rêvées. Ce qui arrive, toutefois, c'est bien le réveil du premier par le second, de telle sorte que le projet se réalise dans l'action et la parole de Vieux-Thomas.

La patrie n'est cependant pas assurée, confortée dans son présent. D'où l'importance du jeu des valeurs sans cesse opposées, qui sont des valeurs de conquête: par l'alliance de l'avant-hier et de l'aujourd'hui, ce sont celles du travail créateur, de la construction, du combat, ce dernier mot recouvrant les premiers : si l'alliance de la mémoire et de la promesse ${ }^{6}$ se fait, le combat sera celui de la réappropriation de soi, de la forêt, de la terre; de la liberté, de la Nature, de l'Histoire; du Destin, si l'on veut. A ces éléments essentiels, ajoutons ce que notre lecture a aussi relevé : une anglophobie marquée (qui a été si caricaturée dans la pièce ${ }^{7}$ ) et un point de vue nettement écologique (contre la consommation, pour la création et la conservation). 
Si l'on met à part cette dernière note, tout cela tinte sans doute d'une façon familière sinon agaçante aux oreilles du lecteur averti : n'est-ce pas là, à quelques détails près, le programme nationaliste tel qu'il traverse toute notre littérature depuis plus d'un siècle? N'est-ce pas là la figure d'un pays dont un parti politique peut maintenant s'inspirer, prenant la relève du discours littéraire ${ }^{8}$ ? Ce roman n'est-il alors que pure répétition du vieux discours national, lui qui va jusqu'à reprendre les refrains et slogans de Menaud? En tout cas, la parution d'un tel texte en 1977 nous invite à faire le point sur la problématique littéraire du pays. Carrier cite Savard qui cite Hémon? Eh bien! reconstituons sommairement la chaîne textuelle dans sa génération graduelle. Partons de Maria Chapdelaine et voyons où cela nous mène, même si nous savons, à l'avance, ne parcourir que quelques grains d'un long chapelet historique.

\section{La chaine textuelle}

On se souviendra qu'à la fin du roman de Louis Hémon, les projets matrimoniaux de Maria, si touchée par la disparition de François Paradis, prennent une tournure définitive, alors que la fille et son père, Samuel, veillent la mère, Laura, qui vient de mourir. Avant de s'assoupir, le vieux fera le récit de sa vie d'éternel défricheur. Puis, laissée à elle-même, Maria songera à son avenir: c'est alors que montent en elle trois "voix": celle de la terre toujours recommencée, de la ville aux mille merveilles et puis celle décisive, "plus grande que les autres" ( $p .187$ ), la " voix du pays de Québec". Aussi, Lorenzo Surprenant, l'émigrant des États, devra-t-il céder la place du «futur» à Eutrope Gagnon. Maria se soumet au misérable destin qui l'attend, résignée, obéissante aux voix des ancêtres, de son père assoupi sinon de la morte même qu'elle veille.

Si vous ouvrez maintenant Menaud maitre-draveur, vous constaterez vite que le récit lui-même s'articule au précédent : vous assistez dès le début, au dialogue d'un père et de sa fille, alors que la mère est morte, depuis un certain temps, toutefois. Tout cela se passant comme si le Samuel abattu, endormi près de sa "défunte", se réveillait sous la plume de Savard, à quelques différences près, dont celle-ci: sa fille opportunément nommée Marie n'est pas à la veille de prendre mari, mais elle est déjá désirée par le Délié que n'aime pas le père. Vous n'êtes donc pas étonné que ressorte de sa bouche de bonne fille, la troisième "voix» qu'entendait Maria. Et vous retrouvez la suite conforme au modèle hémoniste: en butte à "la traîtrise de la chair " dès que le Délié paraît, elle ne s'en soumettra pas moins au devoir du sang et de la race, sous la prédication du père et après la mort tragique de Joson, le Fils en qui le vieux avait mis tous ses espoirs de reconquête du royaume. Elle épousera Alexis le Lucon. Et Menaud aura le sort que l'on sait (la folie).

Ces deux rappels ne constituent en rien un résumé de l'histoire racontée; nous n'avons relevé que les éléments d'articulation et d'embrayage 
narratif servant au texte de Savard. Que fait ensuite Carrier dans // n'y a pas de pays sans grand-père?

Cela commence d'une façon déguisée, inaccessible pour qui ne connaît pas le réseau textuel québécois. Dès l'ouverture narrative, on trouve repris le motif de l'attente du vieux Menaud: Vieux-Thomas est rivé à sa chaise, condamné, comme l'autre à sa fenêtre, à regarder passer les jours, tout en ruminant son impatience de se battre, fût-ce avec la mort. Le lecteur averti s'attend donc à le voir s'éjecter de la berceuse à la manière du draveur qui, excité par la "voix", ouvrait la porte de la maison pour contempler son domaine menacé. Mais Vieux-Thomas ne se lèvera qu'à la fin du récit pour "prendre" l'autobus... En ce sens, le roman de Carrier étend à tout le récit l'action des premières pages de Menaud. Et la mimèse peut dès lors continuer puisque c'est la "voix du pays de Québec" qu'entendra le bûcheron, à travers sa rumination du passé, les événements du présent tels qu'ils se manifestent à lui par le biais de la télévision et dans la voix retrouvée du petitfils emprisonné. Et l'on sait que ce n'est pas tout: le texte de Carrier ira jusqu'à nous faire relire ceux de Hémon et Savard, à la fin du programme que se trace le grand-père qui veille (encore comme Menaud) avant d'aller retrouver Jean-Thomas. Plutôt que de nous arrêter tout de suite aux textes sacrés de la tradition, signalons rapidement d'autres motifs pris aux prédécesseurs. II y a cette reprise fréquente de l'expression a pays de Québec (et non du Québec, comme on le dit plus souvent aujourd'hui). II y a aussi ce que Vieux-Thomas dit toujours: "C'est un pays dur, icitte", formule retouchée de celle de Lorenzo Surprenant, le tentateur de Maria: "lcitte... le pays est trop dur» (136). Plus importante est sûrement la longue marche de VieuxThomas: alors jeune, il s'est engagé tout seul sur les sentiers de ses aînés pour aller aux chantiers de La Tuque. Et, bien évidemment, il "s'écarte", comme Menaud qui suit la "coupe (...) qu'avaient suivie les Anciens dans leur migration vers le Royaume du Saguenay" (p. 195); comme François Paradis qui, lui, avait tenté de rejoindre Maria, partant de La Tuque se dirigeant vers Péribonka, à l'occasion de la Noël. Ainsi, le lecteur québécois a vite fait de se retrouver en pays connu, celui des coureurs de bois, coureurs de pays; et de repérer la géographie du domaine forestier (comme métaphore du pays).

Venons-en maintenant à cette "troisième voix" qu'entend Menaud et que réentend Vieux-Thomas à la veille de son action d'éclat. Tout en continuant notre relevé sommaire des reprises de Carrier, ce sera aussi l'occasion de marquer davantage les écarts du texte, d'élargir notre vue du réseau intertextuel, et de prendre la mesure de l'ambition idéologique du roman.

Vieux-Thomas dira ce qu'il dit toujours: "C'est un pays dur, icitte." Jean-Thomas, selon le rite, ouvrira son livre et il dira: «Écoute, Pépére, cette histoire-ci ressemble à la tienne, c'est l'histoire du pays de Québec: “Nous sommes venus il y a trois cents ans et nous sommes restés... Nous avons marqué un plan du continent nouveau, de Gaspé à Montréal, de Saint-Jean d'Iberville à l'Ungava, en disant: ici toutes les choses que nous avons apportées avec nous, notre culte, notre langue, 
nos vertus et jusqu'à nos faiblesses deviennent des choses sacrées, intangibles et qui devront demeurer jusqu'à la fin. Autour de nous, des étrangers (selon le rite, Jean-Thomas répétera le mot), des étrangers sont venus; ils ont pris presque tout le pouvoir; ils ont pris presque tout l'argent... Et nous nous sommes maintenus, peut-être afin que dans plusieurs siècles encore le monde se tourne vers nous et dise: Ces gens-là sont d'une race qui ne sait pas mourir..." Refermant son livre, Jean-Thomas dira: "Pourriez-vous savoir, Pépére, que Louis Hémon a écrit ça en 1912? " Et ses petites mains fines qui n'ont jamais touché aux travaux rugueux ouvriront un autre livre: "Ce jour-là, assis à sa fenêtre, Menaud écoutait sa fille, Marie, lui lire des passages: “ Nous sommes venus il y a trois cents ans et nous sommes restés... Autour de nous des étrangers sont venus qu'il nous plaît d'appeler des barbares! Ils ont pris presque tout le pouvoir!... (p. 96-97)

Arrêtons-nous là, pour le moment (la citation continue avec la reprise, chez Menaud, de la phrase “ Une race qui ne sait pas mourir » et la mention de l'ouverture de la porte par le draveur). Le récit ne dissimule donc rien de sa dette aux textes antérieurs. II insiste plutôt, voulant rendre hommage à des écrivains qui ont "écrit" notre histoire. Plus encore, le récit de Carrier ritualise ce qui l'était déjà chez Savard: les phrases de Hémon et Savard sont véritablement consacrées comme évangile national. Et de fait, Carrier est davantage fidèle à Savard qu'à Hémon, si l'on veut bien être attentif aux marques de l'écart, puisqu'il ne cite de Hémon que ce qui se trouvait déjà dans Menaud, sans toutefois le restituer fidèlement: ainsi, Jean-Thomas, quand il répète le mot “ étrangers", oublie la suite: "qu'il nous plaît d'appeler des barbares!»

Cette fidélité au texte de Savard réitère chez Carrier l'«oubli» de la deuxième phrase du fameux passage de Maria, où il est dit, dans l'hypothèse d'un éventuel retour des ancêtres français: “... S'il est vrai que nous n'ayons guère appris, assurément nous n'avons rien oublié " (187). Cet aveu d'ignorance, le roman de Hémon a eu bien soin de le souligner auparavant par l'analphabétisme des "habitants", par exemple. Cette carence de la fidélité traditionnelle ne sera pas oubliée par Arsène Bessette, Albert Laberge, Ringuet ${ }^{9}$ et d'autres qui vont écrire après Hémon et avant Savard qui, lui, ne conservera que la revendication du souvenir français; que ce thème de la fidélité, ignorant l'ignorance au profit d'une valorisation religieuse, mystique, du territoire à défendre contre un oppresseur (qui n'est pas plus nommé que chez Hémon, sinon désigné par le mot "barbares"), ses représentants (comme le Délié) et contre les paysans emmaillés dans leurs clôtures (comme Josime). De plus, Savard oubliera tout à fait la deuxième "voix", celle des merveilles urbaines et donnera justement cette vue plutôt méprisante de la première qui chante la terre du cultivateur.

Quant à Carrier, il fait découvrir à un homme condamné par l'âge, la possibilité d'accorder la fidélité ancestrale à l'acquisition du savoir, l'expérience brute mais réfléchie à la conscience politique. Cette dernière reste forcément élémentaire, aux prises avec les incapacités de l'âge et de la 
naïveté de la jeunesse. Un tel schématisme où, par ailleurs, les causes de l'aliénation sont identifiées clairement, l'oppresseur étant toutefois désigné comme économique et anglophone, ne peut que conduire à des moyens d'expression presque folklorique (une manifestation) ou carrément dérisoire (un détournement d'« avion sans ailes" par un vieux étranger au monde réel). II n'en reste pas moins que le choix de la violence est politisé, ce qui évoque des caractéristiques du roman des années soixante. En plus, Carrier revalorise, sans insister toutefois, la "voix" de la terre (ici à " dérocher") comme il fait place à celle de la ville où l'action de reconquête trouve son origine au moins "anecdotique". Car ce n'est que pour mieux revenir au pays profond, terrien et forestier, abandonné par la génération de Dieudonné: cette préférence est marquée dans le privilège accordé à la parole du grand-père, mais elle l'est aussi dans l'importance et le lyrisme des pages consacrées à la lecture des signes sylvestres. Dans tous ces récits de chasse et de pêche, de bûchage et de marche dans les bois, il est difficile de ne pas reconnaître la pédagogie et le testament-manifeste d'Ashini, le héros de Thériault, qui revendique, lui aussi, la liberté d'un territoire et d'un peuple: on y retrouve, sans que Carrier cite le roman de Thériault, les mêmes valeurs du savoir expérimental, de la construction de soi ; et des moyens similaires dans la démonstration narrative, soit l'argumentation fondée sur le mini-conte ou le micro-récit du passé 10.

Mais revenons à l'intertexte carriériste (sans jeu de mots). En plus de la politisation propre au récit du nouveau Menaud, il faut enregistrer d'autres différences. Souvenons-nous d'abord de l'importance accordée par Hémon et Savard à la femme, à la fidèle vestale dont le modèle remonte au moins à Blanche d'Haberville, l'héroïne des Anciens Canadiens 11 qui refuse d'épouser l'«étranger" si fraternel, le conquérant Arché de Locheill, pendant que Jules le vaincu concrétisera son statut en acceptant le mariage des races ennemies. On sait que Maria Chapdelaine et sa réplique savardienne ne font pas autrement que Blanche: derrière le rejet de Lorenzo, le nouvel américain, l'agent de la ville comme dans le rejet du Délié, c'est l'ennemi de la continuité, de la fidélité qui est repoussé.

Or, nous ne retrouvons pas cette trame amoureuse dans la tissure de Carrier. Est-ce parce qu'elle va de soi? Que les femmes de la famille de Vieux-Thomas font leur "devoir"? Sans doute. A défaut de ce fil narratif essentiel aux textes-témoins de l'idéologie nationaliste, on trouve évidemment des personnages féminins, à peine esquissés mais tous les autres personnages restent aussi fantômatiques dans la brumeuse vision du grandpère. Examinons donc brièvement les représentations féminines du récit. II y a d'abord la femme même de Vieux-Thomas: elle a assuré évidemment la "revanche des berceaux ". Elle a, comme la grand-mère qui brodait des fleurs de lys sur les nappes des grands jours, maintenu la chaîne des générations et, la mémoire des origines, encore que l'on ne puisse là-dessus avoir une certitude. Vieux-Thomas se souvient surtout de la célébration des noces d'or, de la mort «assise" de sa propre mère (un homme meurt debout) et de celle, similaire, de son épouse, la Défunte, qu'il ne peut justement pas désigner 
autrement: il a oublié son nom. Alors, le récit fait de même: la femme n'a pas de nom, ni de rôle valorisé, surtout pas la Bru, l'épouse de Dieudonné, la régente, la collectionneuse de portraits de la reine d'Angleterre, la rechigneuse, larmoyante et affaissée comme son mari et ses enfants (à l'exception de Jean-Thomas). La femme est donc ici plus fantômatique que les autres personnages, les mâles: il y a la reine au large sourire de la télévision, la jeune fille innocente ensorcelée par le Diable, ce qui rappelle la Rose Latulippe de L'Influence d'un livre ${ }^{12}$. Reste surtout Justine qui rejette son mari anglophone à l'occasion de sa visite des Fêtes au village natal : la fidélité à la race trouverait là sans doute un exemple convaincant puisqu'elle romprait la fidélité conjugale.

Le choix narratif et thématique est donc clair: l'histoire d'amour racontée ici est encore celle du pays ancestral, mais au sens littéral du pays des pères qui s'autogénèrent, suggérant une sorte de parthénogénèse des mâles, ce qui ne va pas toujours de soi, d'autant plus que la fécondation se fait du grand-père au petit-fils. Tous les fils de Vieux-Thomas sont en effet "stériles", incapables, soumis comme le chauffeur de la Cadillac patronale ou "dérangé» comme Dieudonné qui reproduit à sa manière la folie de Menaud. Épinglons-le, au passage, puisque le récit insiste surtout sur l'échec de ce fils. Le père de Jean-Thomas n'a pu transformer sa révolte en une action à portée collective: il est rentré sur ses "terres" après avoir refusé de perpétuer la servitude héritée, l'endettement héréditaire. II a mis fin à son entreprise de camionnage en faisant une dernière livraison dans la piscine du grand "boss" de Trois-Rivières; et depuis, il quitte la maison dès qu'un problème se pose. Dans l'économie narrative, sa folie naît d'une impuissance: ne pas pouvoir ériger sa conscience malheureuse en analyse, qui se traduirait ultimement dans une action à caractère collectif. Son comportement reste villageois, beauceron: la raison s'en trouve sûrement du côté de son ignorance, puisque son père, une fois informé par Jean-Thomas "comprendra", sans toutefois pouvoir entreprendre une action autre que symbolique; mais le symbole n'est plus celui de l'impuissance, c'est la violence qui est choisie et acceptée en dépit des limites, de l'empêchement, des vicissitudes de l'âge.

Dans ces conditions, tout en maintenant implicitement le rôle traditionnel de la femme, le récit de Carrier dit que la liberté, sa conquête violente, folle ou raisonnée, passe par les hommes, les mâles, sans qu'elle soit pour autant plus assurée comme on l'a vu: la victoire est projetée, programmée mais non réalisée. Nous sommes donc en présence d'un écart considérable par rapport aux textes de référence: comme la folie de Dieudonné juge celle de Menaud (la jauge et la situe) l'absence de la vestale du pays féconde paradoxalement la génération des pères.

Une autre différence remarquable dans le récit de Carrier concerne la place faite à la religion. Chez le vieux bûcheron, nous ne trouvons plus le pouvoir dominant de son institution, telle qu'elle était représentée dans l'histoire de Samuel Chapdelaine. Le curé n'est pas là que l'on consultait sur tous les problèmes matériels et spirituels. N'y est pas non plus la vision 
grandiose, mystique, si accusée dans Menaud, dans les rapports de l'homme à la nature et au pays (que l'on se rappelle la fameuse montagne désignée comme "basilique»). Chez Carrier, le rapport de Vieux-Thomas à Dieu est direct et familier, protestant dirait-on, "charismatique" dirait-on encore. Le grand parlant beauceron s'adresse à Dieu avec la familiarité de Don Camillo, à cette différence près qu'il n'est ni le prêtre italien, ni le prêtre solennel et métaphorique de Savard. II y a là comme une caricature du pouvoir divin, au point où le "cher bon Dieu" peut être l'occasion du détournement phatique que nous avons déjà repéré dans l'humoristique discours du vieux. A la limite, ce "cher bon Dieu" n'est qu'une caution intérieure, que la métaphore bien coulée de la conscience de Vieux-Thomas. Après la disparition de la vestale, c'est le temple lui-même que l'on quitte pour monter dans le car de l'Histoire...

Ainsi, le moins que l'on puisse dire, c'est que la figure accoutumée du pays se condense et se modifie d'un texte à l'autre de notre trio référentiel; de celui du défricheur courageux à celui du draveur mystique au bûcheron mélancolique mais joyeux et entreprenant, entièrement voué au fil et au fils de la liberté, de la continuité nationale, mâle, "machiste" diraient certaines lectrices. En même temps que la figure se déplie et se différencie, elle s'incarne dans un discours typique: du roman réaliste (et poétique), on est passé à la poésie épique pour en arriver à la parole familière du conte. L'impression première de la répétition peut persister si l'on s'arrête à la carence réaliste du discours de Carrier. Son choix d'une parole nostalgique, onirique et schizoïde, traversée graduellement par les échos du réel, ne peut que construire une autre image rêvée sinon figée du pays: celui que l'on sait passant sur le pont de deux générations, celle d'avant-hier, forte justement de ses rêves, de sa fidélité et celle de demain, le presque aujourd'hui, aussi fragile que politisée. Est-ce pour cela que surgit une question, posée dans le texte lui-même, à savoir que notre imagination romanesque contemporaine n'a que peu de prises sur le présent immédiat et que dès lors le pays, dans sa représentation littéraire, reste dans les limbes de la fiction, toujours aussi incertain, selon la formule de Ferron?

\section{Au bout du conte: une Grande Histoire}

En ce cas, nous sommes ramené aux fictions des années soixante. Estce pour cela qu'à la suite de la longue citation de Savard-Hémon, le narrateur et porte-parole du grand-père déclare:

Jean-Thomas refermera le livre et il dira : "Félix-Antoine Savard a écrit ces mots-là en 1929; maintenant, qu'est-ce qu'on va écrire dans les livres faits au pays de Québec?" Ecoutant lire son petit-fils, VieuxThomas maintenant comprendra [...] saura que l'heure est venue de prêter, de donner ses oreilles à une Grande Histoire" (98).

Qu'est-ce qu'on va écrire dit le porte-parole de l'auteur sinon l'Histoire elle-même? Non pas le roman historique: les majuscules de la "Grande Histoire" renvoient à ce que Malraux appelait "se donner un Destin". Cela 
n'est plus affaire de fiction dit le texte de Carrier qui oppose les petites histoires à la Grande, pour laquelle on ne prête pas mais on donne ses oreilles : derrière la drôlerie, il y a bien le don, le sacrifice, l'effort personnel à faire.

Ainsi, cette entreprise n'est pas la pure et simple itération de l'évangile de Louis et de Félix-Antoine: des “contes du pays incertain" nous voilà entrainés dans la problématique du faire historique, telle qu'elle se pose à la fin du Couteau sur la table de J. Godbout et de Prochain épisode de Hubert Aquin. Le couteau du vieux sourd et la mention de l'heure $\mathrm{H}$ (pour historique) sont des motifs qui ne relèvent pas de la répétition hagiographique de Savard et Hémon. Dira-t-on alors que Carrier répète aussi Godbout et Aquin ? Oui, d'une certaine façon et par choix. Ecrivant en 1977, il donne la parole à un vieillard des années soixante (d'où ce recours à un manifestant du "samedi de la matraque" (10 octobre 1964)) et tente une synthèse de notre discours national de Maria Chapdelaine à Prochain épisode. Ce geste d'écriture porte une signification précise: "Au pays de Québec, rien n'a changé "... depuis le milieu des années soixante ${ }^{13}$.

Au bout du conte, une grande histoire: voilà où nous conduit provisoirement notre analyse. Depuis le titre jusqu'aux derniers mots; depuis le va-et-vient de la berçante jusqu'à la camisole de force; depuis la complainte écologique jusqu'au chant de la réconciliation des générations mâles et jusqu'au choix de l'action libératrice, c'est une lecture de la figure du pays qui se propose dans la narration de Carrier.

Le mot "pays" circule ici en toute liberté sémantique, dans une utilisation optimale: c'est la patrie, petite et grande, l'une fondant l'autre; c'est le domaine et le royaume de ce terrain littéraire où elle vient s'opposer a un Etat rejeté et combattu, la notion d'appartenance ne se conjuguant pas avec elle de l'autorité reconnue, établie. Dans cette thématique du lit défait, comme le disait Hubert Aquin dans un article ${ }^{14}$ et comme on la trouve reprise dans la brève histoire de Justine, le seul mariage qui tienne est celui du faire et de la connaissance, de la nature et de la culture. Et l' « impossible fête" dont parlait André Brochu à propos de Menaud ${ }^{15}$ est déjà vécue ici dans l'imagination et le discours.

A partir du réseau textuel annoncé, avoué et sur-souligné dans le récit de Carrier, c'est tout l'intertexte national qui est convoqué. II nous reste donc à contrôler notre repérage, en retournant aux "contes du pays incertain " (1960-1963), aux romans de l'époque de Parti pris (1963-1968) et à ceux qui sont venus depuis. Car notre lecture d'une lecture devient l'hypothèse à vérifier: il y aura beaucoup à faire s'il est vrai que le recours au pays imaginaire va de soi quand le pays réel (l'État) fait défaut ${ }^{16}$. 
1. Aux Éditions internationales Alain Stanké, comme Les enfants du bonhomme la lune (contes), paru en 1979. Les autres titres énumérés ont été publiés aux Éditions du Jour.

2. Annonce idéologique: ce qui s'affiche, se placarde en couverture; ce qui est "préfiguré» dans le conditionnement du livre: le message de l'emballage éditorial.

3. Notre démarche vise à isoler les propositions "qui, dans le corps du récit, ont l'aspect de règles ou de vérités générales", comme le dit Jacques Dubois dans son essai, L'Assommoir d'Émile Zola, société, discours, idéologie, Paris, Larousse, "Thèmes et textes", 1973. Voir les pages 105 et 106 où il définit ses « indicateurs": le métatexte (titre et préface), l'incipit, les propositions et les documents extérieurs, intertextuels.

4. Revue québécoise qui a paru de 1970 à 1978.

5. Bessette, Gérard, Les anthropoïdes, Montréal, La Presse, 1977.

6. Selon la belle formule de N. Kattan (La mémoire et la promesse, Hurtubise-HMH, Montréal 1978.

7. L'adaptation du roman a été jouée au théâtre Port-Royal, à Montréal, du 2 mars au 2 avril 1978.

8. "Avec le PQ, les mots sont au pouvoir", titre le Devoir du 26 avril dernier (p. 21) d'après une dépêche de la Presse canadienne qui signale qu'«au moins 13 membres du parti au pouvoir ont au moins un livre à leur crédit». Le méme paragraphe cite Roch Carrier: “Autrefois, la littérature québécoise s'écrivait dans l'Opposition; elle revendiquait, et - plus ou moins ouvertement - elle dénonçait. Mais depuis que le parti Québécois est au pouvoir, c'est plutỏ́t comme si la littérature était au pouvoir". La dépêche relève aussi les mots « cruciaux»: "souveraineté-association", "fédéralisme renouvelé ", "séparatiste", "indépendance ${ }^{\prime} . .$.

9. Je pense au Débutant (1914), à La scouine (1918) et à Trente arpents (1938).

10. Voir Ashini, Montréal, Fides 1960.

11. Aubert de Gaspé père, P., Les anciens Canadiens, Québec, Desbarats et Derbishire, 1863.

12. Aubert de Gaspé fils, P., L'influence d'un livre, roman historique, Québec, Cowan et fils, 1837.

13. Ou encore : quand la littérature est au pouvoir, on ne peut que faire des reprises, une synthèse du déjà-dit... (voir supra, note 8 ).

14. Aquin, $H_{\text {., }}$ "Le corps mystique", Parti pris, vol. I, no 5, février 1964, p. 30-36.

15. Brochu, A., « Menaud ou l'impossible fêtè, L'Instance critique, Montréal, Leméac, "Indépendances", p. 247-274.

16. Ce texte est extrait d'un essai en préparation. 